\title{
Uso de pruebas afectivas, discriminatorias y descriptivas de evaluación sensorial en el campo gastronómico
}

Use of affective, discriminatory and descriptive sensory evaluation tests in the gastronomic field

Uso de testes afetivos, discriminatórios e descritivos de avaliação sensorial no campo gastronômico

\author{
Norma V. Cárdenas-Mazón ${ }^{\mathrm{I}}$ \\ nocardenas@espoch.edu.ec \\ Carlos E. Cevallos-Hermida II \\ ccevallos@espoch.edu.ec \\ Juan C. Salazar-Yacelga III \\ j_salazar@espoch.edu.ec \\ Efraín R. Romero-Machado ${ }^{\mathrm{IV}}$ \\ eromero@espoch.edu.ec \\ Patricia L. Gallegos-Murillo V \\ p_gallegos@espoch.edu.ec \\ Mayra E. Cáceres-Mena VI \\ mecaceres@espoch.edu.ec
}

Recibido: 10 de enero de 2018 * Corregido: 18 de marzo de 2018 * Aceptado: 11 de junio de 2018

I. Magister en Nutrición Infantil, Doctora en Nutrición y Dietética, Docente de la Escuela Superior Politécnica de Chimborazo, Riobamba, Ecuador.

II. Magister en Procesamiento de Alimentos, Licenciado en Gestión Gastronómica, Docente de la Escuela Superior Politécnica de Chimborazo, Riobamba, Ecuador.

III. Magister en Procesamiento de Alimentos, Licenciado en Gestión Gastronómica, Docente de la Escuela Superior Politécnica de Chimborazo, Riobamba, Ecuador.

Iv. Magister en Procesamiento de Alimentos, Licenciado en Gestión Gastronómica, Técnico Superior en Gastronomía, Docente de la Escuela Superior Politécnica de Chimborazo, Riobamba, Ecuador.

v. Magister en Informática Educativa, Ingeniero en Computación y Ciencias de la Informática, Tecnólogo en Análisis de Sistemas, Técnico Superior en Procesamiento Electrónico de Datos, Analista de Sistemas de Computación, Docente de la Escuela Superior Politécnica de Chimborazo, Riobamba, Ecuador.

vi. Magister en Matemática Básica, Magister en Desarrollo de la Inteligencia y Educación, Doctora en Matemática, Docente de la Escuela Superior Politécnica de Chimborazo, Riobamba, Ecuador. 


\section{Resumen}

Evaluación sensorial, disciplina científica utilizada para evocar, medir, analizar e interpretar las reacciones humanas a aquellas características de los alimentos y materiales que son percibidos a través de los sentidos de la vista, oído, olfato, gusto y tacto. En tal sentido, se presenta una propuesta teórica para el uso de pruebas afectivas, discriminatorias y descriptivas de evaluación sensorial en el campo gastronómico. Entre los materiales y métodos propuestos se indican el panel de degustación individual, los escupideros (tarrinas de plástico con tapa), vasos plásticos pequeños y grandes, servilletas, manteles, cubre manteles y cubre sillas, taller de degustación, yogurt de tres sabores de dos plantas lácteas distintas, vino tinto, vino Blanco, agua natural y chocolate de dos empresas distintas, para su evaluación se utilizaran dos tipos de metodología una de tipo sensorial de alimentos y bebidas, y la otra de tipo analítica y como instrumento tres fichas a saber; un modelo de prueba descriptiva de evaluación sensorial a utilizar para la degustación de yogurt, un modelo de ficha para cata de base de prueba discriminativa de evaluación sensorial utilizada para la degustación de vinos y un modelo de ficha para aceptabilidad de chocolate. Entre sus conclusiones se pudo determinar que las pruebas afectivas han de usar degustadores no experimentados, mientras las descriptivas y discriminatorias se apoyaran en profesionales en el campo de la gastronomía relacionados con el procesamiento y elaboración de los productos degustados, cuyo análisis incluirá la escala hedónica y el análisis por varianza.

Palabras clave: evaluación sensorial; prueba discriminativa; pruebas afectivas y pruebas descriptivas.

\section{Abstract}

Sensory evaluation, scientific discipline used to evoke, measure, analyze, and interpret human reactions to those features of food and materials that are perceived through the senses of sight, hearing, smell, taste and touch. In this sense, is a theoretical proposal for the use of affective, discriminatory and descriptive tests sensory evaluation in the gastronomic field. Between materials and proposed methods are indicated individual tasting panel, the escupideros (plastic Lidded tubs), small and large plastic glasses, napkins, tablecloths, tablecloths covered and covered chairs, wine tasting workshop, yogurt three flavors of two different dairy plants, red wine, white wine, natural water and chocolate in two different companies, for evaluation were used two types of methodology a sensory type of food 
and drinks, and the other of analytical type and as instrument three chips namely; a descriptive test of sensory evaluation model to use to the taste of yogurt, a model sheet for tasting of basis of sensory discriminative test used for wine tasting and a model sheet for acceptability of chocolate. Among its conclusions it was determined that affective tests must use tasters not experienced, while the descriptive and discriminatory will be supported in professionals in the field of gastronomy with the processing and production of the tasted products, whose analysis will include the hedonic scale and analysis by variance.

Keywords: sensory evaluation; affective tests; descriptive tests and discriminatory tests.

\section{Resumo}

Avaliação sensorial, a disciplina científica utilizada para evocar, medir, analisar e interpretar as características humanas aos de alimentos e materiais que são percebidos através dos sentidos da visão, audição, olfato, paladar e tato reações. A este respeito, uma proposta teórica para o uso de afetiva, testes de avaliação sensorial discriminatórias e descritivos no campo da culinária é apresentada. Entre os materiais e métodos propostos degustação painel individual, os escupideros (tubos de plástico com tampa), pequenas e grandes copos de plástico, guardanapos, toalhas de mesa, diagramas tampas e coberturas de cadeira, oficina degustação iogurte três sabores dois indicado várias fábricas de lacticínios, vinho tinto, vinho branco, água mineral e chocolate em duas empresas diferentes, para avaliação de dois tipos de metodologia do tipo sensorial de alimentos e bebidas e outro tipo analítico e como um instrumento três guias foram usadas para saber ; um modelo de teste de avaliação sensorial descritiva para ser utilizada para a degustação iogurte uma folha de modelo para teste discriminativo base de vinho avaliação sensorial utilizada para prova de vinhos e de uma forma que dá para a aceitabilidade do chocolate. Entre as suas conclusões foi determinado que os testes afetivos têm que usar provadores inexperientes, enquanto descritivo e discriminatória vai contar com profissionais da área da gastronomia relacionada com o processamento e preparação de produtos amostrados, a análise incluirá escala hedônica a análise por variância.

Palavras chave: avaliação sensorial; teste discriminativo; testes afetivos e testes descritivos. 


\section{Introducción}

La evaluación sensorial de los alimentos, constituye hoy en día un pilar fundamental para el diseño y desarrollo de nuevos productos alimenticios. Sin duda, el poder medir en el laboratorio el grado de satisfacción que brindará un determinado producto, nos permite anticipar la aceptabilidad que éste tendrá. La evaluación sensorial, para Asunción (2009), es también un elemento necesario para desarrollar una estrategia de marketing, ya que el placer o satisfacción sensorial o prueba hedónica es una determinante importante del consumo de alimentos.

Desde hace bastante tiempo se ha aplicado la Evaluación Sensorial sin base científica en la industria de alimentos. Se trata, según Díaz (2013) de exámenes organolépticos- especializados, habitualmente usados en bebidas estimulantes. Se ha logrado una certeza sorprendente con los catadores de vinos, que pueden llegar a establecer la zona, viña y año de producción. También se conocen resultados exitosos obtenidos por los catadores de cerveza, té, café y yerba mate.

El vino es una bebida muy compleja por su composición, y los factores naturales y humanos que influyen en sus características, por su constitución bioquímica y química que hacen de él un producto especial y atrayente. Es conocido que los principales factores que influyen sobre las calidades del vino son cuatro: el suelo, la variedad de cepa, el clima y el hombre.

La degustación o cata, es una operación en la se tiene que coordinar un complejo mecanismo de estímulos que, al involucrar los sentidos humanos, origina diferentes sensaciones: el reconocimiento y la interpretación de las sensaciones se designan con el término percepción. La cata de un vino se lleva a cabo a través de la vista, el olfato, el gusto y el tacto; cada evaluación general es irremplazable, para dar juicio global, que desde la evaluación cualitativa permite obtener implicaciones técnicas.

La cata constituye un momento de fundamental importancia para comprobar las características de un vino. Permite para Ratti (2011) analizar los componentes, evaluarlos uno a uno, juzgarlos en conjunto, apreciar sus características positivas, señalar los caracteres negativos; la degustación es una operación indispensable para orientar la producción del vino, no se la puede pasar por alto ya que no solo permite la evaluación del producto sino también permite un juicio técnico no reemplazable si es efectuada por un catador que tenga conocimiento de las conexiones profundas entre constituyentes del vino y las sensaciones que origina. 
Catar el chocolate puede ser un placer para los 5 sentidos. La temperatura ideal para conservar el chocolate está entre $14^{\circ}$ y $18^{\circ} \mathrm{C}$ y una humedad del $57 \%$, pero la temperatura óptima para catar el chocolate oscila entre 19 y $25^{\circ} \mathrm{C}$ por lo que en algunas ocasiones es necesario acondicionar el producto al menos una hora antes de llevar a cabo la degustación.

Para degustar el Yogurt se pueden aplicar dos tipos de pruebas, las afectivas en las que se pueden seleccionar a degustadores al azar que expresen su grado de aceptación del producto con un criterio me gusta / no me gusta; sin embargo, se puede aplicar, un análisis discriminativo en el que se evalúe mediante una comparación múltiple escalar no estructurada con un panel de degustadores entrenado en criterios de evaluación de este tipo de producto que son los descriptores: sabor (ácido - dulzor), color (uniforme - blanco - brillo o intenso), olor (natural ácido - fresco agradable), consistencia (firmeza - cremosidad - cuerpo - homogeneidad - grumosidad), apariencia (homogeneidad - firmeza - cremosidad).

Sin embargo, en investigaciones que han aplicado el test de evaluación sensorial como es el caso de la "Elaboración de requesón de leche de cabra con Nisina como conservante natural", en la que se realizaron tres tipos de análisis; Bromatológico, en el que se investigó Grasa, Proteína, ceniza y Humedad. Microbiológico, Staphyloccocus aureus, Echericha coli, Salmonella SPP, Coliformes Totales. Análisis Organoléptico o Pruebas Afectivas: Color, Aroma, Sabor, Textura, se pudo constatar que el requesón elaborado con leche de cabra en promedio registro 59.95\% y un coeficiente de variación de $0.92 \%$, al realizar el respectivo análisis de varianza, no se identificó diferencias estadísticas entre los tratamientos, por lo que se puede mencionar que la Nisina no interviene en la humedad del requesón. Asimismo, la evaluación sensorial mostraron que el color del requesón de leche de cabra al utilizar $0.6 \%$ de Nisina se registró $4.73 / 5$ puntos, que representa el 94.6\%, corresponde a un color agradable según los catadores, a pesar de no registrar diferencias estadísticas entre los diferentes tratamientos supero a los niveles extremos puesto que alcanzaron una menor calificación según los degustadores, principalmente de producto con el tratamiento control que apenas llego a registrar 4.37/5.0 puntos, representa el87.4\% que a pesar de ser muy bueno no llega a ser excelente que es lo que requiere el consumidor de este tipo de productos. Con base en lo expuesto, se presenta en este artículo una propuesta teórica para el uso de pruebas afectivas, discriminatorias y descriptivas de evaluación sensorial en el campo gastronómico. 


\section{Pruebas degustativas}

Las pruebas degustativas son naturales en el hombre, porque desde que prueba un producto empieza a hacer juicios sobre él, si le gusta o le disgusta, o describe sus características propias de sabor, olor, textura, etc. Es una herramienta básica cada vez más usada en la alimentación, que si no se la sabe utilizar puede convertirse en la herramienta con mayor sesgo en el resultado.

La evaluación sensorial. Es aquella, de acuerdo a Wittig (2001) que usa técnicas basadas en la fisiología y psicología de la percepción. Las pruebas sensoriales llevan a cabo varias pruebas según sea la finalidad para la que se efectúe. Existen tres tipos de prueba, las afectivas, discriminatorias o discriminativas y descriptivas; en las que se busca conformar un panel de análisis sensorial. Cabe destacar, que varias pruebas pueden ser utilizadas para identificar la apreciación del catador sobre los alimentos, sustancias, o preparaciones que degusta, así se pueden mencionar las pruebas afectivas, las pruebas discriminatorias y las pruebas descriptivas.

Las pruebas afectivas. Refieren a aquellas en las cuales el juez expresa su reacción subjetiva del producto, indicando si le gusta o si prefiere otro. Por lo general se realizan con paneles inexpertos o con solamente consumidores. Entre las pruebas afectivas están las de medición del grado de satisfacción y las de aceptación.

Las pruebas discriminatorias. Aquellas que no requieren conocer la sensación subjetiva que provoca un alimento, se busca establecer si hay diferencia o no entre dos o más muestras, y en algunos casos la magnitud o importancia de esa diferencia. Las pruebas discriminativas más usadas son las pruebas de comparación apareada simple, triangular, duo - trío, comparaciones múltiples y de ordenamiento.

Las pruebas descriptivas. Refieren aquellas pruebas donde el juez establece los descriptores que definen las características sensoriales de un producto y así cuantifican las diferencias existentes entre varios productos. Consiste en describir el color y el sabor integral de un producto, así como sus atributos individuales. A través de estas pruebas se define el orden de aparición de cada atributo, grado de intensidad de cada uno, sabor residual y amplitud o impresión general del sabor y el olor. 


\section{Materiales y Métodos}

\section{Materiales:}

Panel de degustación individual

Escupideros (tarrinas de plástico con tapa)

Vasos plásticos pequeños y grandes

Servilletas

Manteles, cubre manteles y cubre sillas

Taller de degustación (adaptado)

Yogurt de tres sabores de dos plantas lácteas distintas

Vino tinto

Vino Blanco

Agua natural

Chocolate de dos empresas distintas

Requesón de leche de cabra con Nisina en tres niveles: $0,4-0,6$ y 0,8

Métodos: Se va a utilizar dos tipos de metodología usada para la evaluación sensorial de alimentos y bebidas, la metodología afectiva (Pruebas Afectivas) y la metodología analítica (Pruebas Discriminativas - Pruebas Descriptivas) (Ver anexos 1, 2 y 3).

Uso de Pruebas afectivas: Estas pruebas miden la probable reacción del consumidor con respecto a un nuevo producto, para este tipo de test no se requiere de degustadores especializados por lo que se usa frecuentemente como un análisis de aceptabilidad de uno o más productos. En una prueba de aceptabilidad se pueden utilizar hasta 5 muestras que no generen fatiga en los receptores gustatorios. La hora a la que se da la evaluación también es muy importante, no debe acercarse nunca a la hora de la comida ya que generará una respuesta poco confiable.

Uso de pruebas discriminatorias: Permiten comparar dos o más productos, e incluso estimar el tamaño de la diferencia. De manera general son sencillas y de gran utilidad práctica, sin embargo, no hay que descartar que para la realización de esta prueba se requiere de jueces altamente calificados. Este tipo de test proporciona la información más completa sobre los caracteres sensoriales de las muestras, ya que mide los efectos de cada una de las características de calidad sobre la complejidad del total. 
Pruebas descriptivas: por medio de este test es posible evaluar hasta seis muestras diferentes. Usa un panel que no necesariamente está entrenado. Las muestras se valoran de acuerdo a una escala numérica de calidad que va de "Excelente" a "Malo", e una puntuación de 1 a 9, y se pide al degustador que marque en ella la calidad de las muestras que le presentan para evaluar.

\section{Resultados y Discusión}

Posterior al análisis de la información, fue posible considerar que los resultados de la aplicación de las pruebas descriptas se obtendrán de tres grupos de degustadores a saber:

Para las Pruebas afectivas: nos apoyaremos en un grupo de degustadores no experimentados para la determinación de las pruebas afectivas que tengan relación con el campo gastronómico, mientras que para las pruebas descriptivas como para las discriminatorias nos apoyamos en profesionales en el campo de la gastronomía relacionados con el procesamiento y elaboración de los productos degustados.

Análisis Estadístico: Para el análisis descriptivo se usa la escala hedónica completa o simplificada de evaluación, los resultados del panel se analizan por varianza, pero también pueden transformarse en ranking y analizar por cómputos.

Para las pruebas discriminatorias: la degustación se la realzará con degustadores experimentados, representantes de la Asociación Nacional de Chefs del Ecuador.

Análisis Estadístico: Debido a que estos métodos son frecuentemente utilizados como herramientas de control de calidad, los resultados se analizan estadísticamente planteando la "hipótesis nula" y la “hipótesis alternativa”. Es decir, se plantea hipótesis de que las muestras no difieren entre sí, o lo que es lo mismo, que no se detectan diferencias.

Para las pruebas descriptivas: nos apoyaremos con los asistentes al taller que tengan nociones de Evaluación Sensorial de alimentos y bebidas.

Análisis estadístico: La evaluación estadística se la hace en base a juicios favorables para cada calificación (cómputos). 


\section{Conclusiones}

Las pruebas afectivas han de usar degustadores no experimentados, mientras las descriptivas y discriminatorias se apoyan en profesionales en el campo de la gastronomía relacionados con el procesamiento y elaboración de los productos degustados, cuyo análisis incluirá la escala hedónica y el análisis por varianza.

Se solicitarán los servicios de degustadores adscritos a la Asociación Nacional de Chefs del Ecuador, en el caso de las pruebas discriminatorias y su análisis estadístico se hará bajo el planteamiento de pruebas hipotéticas nulas y alternativas.

Las pruebas descriptivas se harán con los asistentes al taller que tengan nociones de Evaluación Sensorial de alimentos y bebidas y su análisis se hará con base a juicios favorables para cada calificación.

\section{Referencias Bibliográficas}

Alvarado, C. Coronado, M. Prosperi, F. Guerra, M. (2011). Desarrollo de yogurt con capacidad antioxidante elaborado con leche de cabra (Capra hircus) y tomate de árbol (Cyphomandra betacea Sendtn). Revista Venezolana de Ciencia y Tecnología de Alimentos. Universidad Central de Venezuela, Facultad de Ciencias Veterinarias - Universidad Simón Bolívar, Departamento de $\begin{array}{lll}\text { Procesos Biológicos } & \text { y } & \text { Bioquímicos. }\end{array}$ http://www.rvcta.org/Publicaciones/Vol2Num2/ArchivosV2N2/Alvarado-Carrasco_et_al._RVCTAV2N2.pdf Venezuela. Julio - Diciembre.

Díaz, R (2013). Evaluación sensorial. Edición digital

Ratti, R (2011). Cómo degustar los vinos. Manual del catador. Ediciones Mundi - Prensa. 2da Edición. Madrid, México. 2011

Wittig, E (2001). Evaluación sensorial. Una Metodología actual para la tecnología de alimentos. Edición digital 


\section{Anexos:}

Anexo 1. Modelo de ficha de Prueba Descriptiva de Evaluación Sensorial utilizada para la degustación de yogurt

Tipo: Valoración

Test: Descriptivo

Producto:

\section{Nombre:}

\section{Fecha:}

Hora:

Por favor, califique la calidad de las muestras que se presentan de acuerdo a la escala de calidad que se indica. Marque sólo una calificación por muestra.

\begin{tabular}{|c|c|c|c|c|c|c|c|c|c|}
\hline \multicolumn{3}{|c|}{ Muestra $N^{\circ}$} & \multicolumn{3}{|c|}{ Muestra $\mathbf{N}^{\circ}$} & \multicolumn{2}{|c|}{ Muestra $\mathbf{N}^{\circ}$} & \multicolumn{2}{|c|}{ Muestra $\mathbf{N}^{\circ}$} \\
\hline ( & 1 & excelente & ( & 1 & excelente & $(1)$ & excelente & ( & excelente \\
\hline 1 & 1 & bueno & 1 & 1 & bueno & ) & bueno & ( & bueno \\
\hline ( & 1 & regular & ( & 1 & regular & 1 & regular & ( & regular \\
\hline ( & ) & malo & ( & ) & malo & $(\quad)$ & malo & ( & malo \\
\hline
\end{tabular}

Anexo 2. Modelo de ficha para Cata de Base de Prueba Discriminativa de Evaluación Sensorial utilizada para la degustación de vinos

Muestra $\mathrm{N}^{\circ}$

Vino.

Añada. 
Uso de pruebas afectivas, discriminatorias y descriptivas de evaluación sensorial en el campo

\begin{tabular}{|c|c|c|c|c|c|}
\hline \multirow[b]{2}{*}{ Sensaciones } & \multirow[b]{2}{*}{ Referencia } & \multicolumn{3}{|c|}{$\begin{array}{c}\text { Cruzar la casilla } \\
\text { correspondiente al } \\
\text { inicio }\end{array}$} & \multirow[b]{2}{*}{ OBSERVACIONES } \\
\hline & & 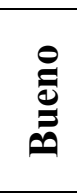 & 递 & : & \\
\hline Visuales & $\begin{array}{l}\text { Color } \\
\text { Limpidez }\end{array}$ & & & & \\
\hline Olfativas & $\begin{array}{l}\text { Franqueza } \\
\text { intensidad }\end{array}$ & & & & \\
\hline Gustativas & $\begin{array}{l}\text { Franqueza } \\
\text { intensidad }\end{array}$ & & & & \\
\hline $\begin{array}{l}\text { Gusto - } \\
\text { olfativas }\end{array}$ & $\begin{array}{l}\text { Franqueza } \\
\text { intensidad }\end{array}$ & & & & \\
\hline \multicolumn{2}{|c|}{ Armonía } & & & & \\
\hline
\end{tabular}

\section{JUICIO}

Fecha

Lugar

El catador

Anexo 3. Modelo de ficha para Aceptabilidad de chocolate

Escala Hedónica simplificada de Evaluación Sensorial

\begin{tabular}{|l|l|l|}
\hline \multicolumn{1}{|c|}{ categoría } & \multicolumn{2}{c|}{ indicador } \\
\hline sabor & me gusta & no me gusta \\
\hline color & & \\
\hline aroma & & \\
\hline textura & & \\
\hline consistencia & & \\
\hline
\end{tabular}

